\title{
Genetic association between direct and indirect measures of body energy in dairy cows
}

G Banos ${ }^{1}$, M P Coffey ${ }^{2}$

${ }^{1}$ Department of Animal Production, Faculty of Veterinary Medicine, Aristotle University of Thessaloniki, Thessaloniki, Greece, ${ }^{2}$ Sustainable Livestock Systems Group, Scottish Agricultural College, Edinburgh, United Kingdom

Email:banos@vet.auth.gr

Introduction Body energy refers to the amount of internal energy a cow has at her disposal to support physiological functions (milk production, growth, reproduction, maintenance, activity). Ideally, body energy would be directly measured using individual feed intake and physiological function records. In field conditions, however, such information is not available. For this reason, indirect measures of body energy have been suggested based on the body condition score and live weight of a cow. The usefulness of these traits depends on their true association with direct body energy. The objective of this study was to derive the correlation between direct and indirect measures of body energy in dairy cows.

Materials and methods Individual records for milk yield and composition, dry matter intake, live weight, and body condition score (BCS) were extracted for $1^{\text {st }}$ lactation Holstein cows from the Scottish Agricultural College database of records gathered at the research station at Langhill Farm. Cows had calved between 1990 and 2005, and participated in feed and selection experiments. Milk yield records were available almost daily (5-7 days/week), dry matter intake was recorded 3 times/week, and milk composition, live weight and BCS were available on a weekly basis. Daily phenotypic records were calculated for each cow and trait using a mixed model that included the fixed effects of feeding and genetic group, year-bymonth of record, year-by-season of calving, age at calving, and $3^{\text {rd }}$ order orthogonal polynomial of days in milk; an interaction of the latter with cow was also fitted as a random effect. Solutions were used to calculate daily records for each trait. At the end of this step, there were 246,708 daily records for 801 cows. Subsequently, a direct measure of body energy was calculated based on all trait records plus data on the chemical composition of feed. The effective energy system proposed by Emmans (1994) was used. In addition, 3 indirect measures of body energy were considered: BCS, energy content (EC) and cumulative effective energy (CEE), the last two being combinations of BCS with live weight. Banos and Coffey (') describe these direct and indirect traits in detail. The genetic correlation of each indirect trait with direct body energy was calculated using a bivariate random regression model that included the same fixed effects as the previous model, in addition to a cow genetic and a permanent environment random effect. This analysis yielded genetic correlation estimates for each day of lactation. Finally, the possibility to predict direct body energy from each indirect measure was assessed with a model including the same fixed effects plus a regression on each indirect measure. For this reason, the dataset was randomly split into 3 equally sized subsets and, in each of 3 permutations, 2 subsets were used to calculate the regression coefficients while the $3^{\text {rd }}$ subset was used to implement and compare predicted and observed direct body energy on independent data. Comparison criteria were the mean absolute difference, root mean square error, product moment correlation and an accuracy indicator based on the prediction error variance.

Results Significant $(\mathrm{P}<0.05)$ genetic correlations between direct body energy and BCS were observed on days 21-107 of lactation and ranged from 0.41 to 0.60 . For EC and CEE, corresponding results were days 18-101 and 32-122 of lactation and correlation estimates of $0.38-0.54$ and $0.45-0.70$, respectively. Strongest genetic correlations with direct body energy were found on days 61, 60 and 71 for BCS, EC and CEE, respectively. Comparisons of observed and predicted direct body energy from indirect traits (BCS, EC and CEE) are shown in Table 1. These results are averages of the 3 permutations and pertain to days of lactation with a significant genetic correlation between direct and indirect body energy measures. All estimates in Table 1 were significantly greater than zero $(\mathrm{P}<0.05)$. No statistically significant differences were found in the predictive ability of the three indirect measures.

Table 1 Comparison between observed direct body energy and predicted direct body energy from 3 indirect traits

\begin{tabular}{lllll}
\hline \hline Predictor trait & $\begin{array}{l}\text { Mean absolute } \\
\text { difference }(\mathrm{MJ})\end{array}$ & $\begin{array}{l}\text { Root mean square } \\
\text { error (MJ) }\end{array}$ & Correlation (\%) & Accuracy (\%) \\
\hline Body condition score & 14.84 & 18.97 & 89.02 & 82.74 \\
Energy content & 15.26 & 19.49 & 88.52 & 82.14 \\
Cumulative effective energy & 14.48 & 18.51 & 89.22 & 82.99 \\
\hline \hline
\end{tabular}

Conclusions This study revealed significant positive genetic correlations between direct and indirect body energy measures, especially in the first 2-3 months of lactation. These indirect body energy measures may be used to predict, with relative accuracy, direct body energy. Differences among the three indirect body energy traits were negligible. Because of its simplicity in recording at field conditions, body condition scored at the time of the $2^{\text {nd }}$ or $3^{\text {rd }}$ milk test is recommended as a proxy to direct body energy at that stage of lactation.

Acknowledgements This work was financed by a grant from the Scottish government. Data were made available by the Langhill Farm, Scotland. Ross McGinn is acknowledged for maintaining the database and managing the process.

\section{References}

Banos, G. and Coffey, M.P. 2010. Animal 4, 189-199.

Emmans, G.C. 1994. British Journal of Nutrition 71, 801-821. 